Disponível em:

http://editora.unoesc.edu.br/index.php/race

RACE, Joaçaba, v. 16, n. 2, p. 729-756, maio/ago. 2017

\title{
DETERMINANTES DA DIVULGAÇÃO DE INFORMAÇÕES DE RISCO DE MERCADO POR EMPRESAS NÃO FINANCEIRAS
}

Determinants of disclosure of market risk information by non-financial companies

Benedito Manoel do Nascimento Costa

E-mail: beneditocosta50@gmail.com

Mestre em Administração e Controladoria pela Universidade Federal do Ceará; Especialista em Contabilidade Gerencial pela Universidade do Estado do Rio Grande do Norte;

Professor Assistente no Departamento de Ciências Contábeis da Universidade do

Estado do Rio Grande do Norte. Endereço para contato: Avenida Lauro Maia, 782, Estação, 59770-000, Patu, Rio Grande do Norte, Brasil.

Paulo Henrique Leal

E-mail: pa.henriqueleal@gmail.com Mestre em Administração e Controladoria pela Universidade Federal do Ceará; Graduado em Ciências Contábeis pela Faculdade de Juazeiro do Norte; Professor Assistente no Curso de Ciências Contábeis da Universidade Federal do Sul e Sudeste do Pará.

Vera Maria Rodrigues Ponte E-mail: vponte@fortalnet.com.br Doutora em Controladoria e Contabilidade pela Universidade de São Paulo; Especialista em Finanças pela Universidade Federal do Ceará; Professora Associada I no Programa de Pós-graduação em Administração e Controladoria da Universidade Federal do Ceará.

Artigo recebido em 18 de setembro de 2016. Aceito em 04 de abril de 2017. 
Resumo

Neste estudo teve-se como objetivo identificar os fatores determinantes do nível de observância de empresas não financeiras às exigências de divulgação definidas no CPC 40 (R1). Nesse sentido, foram analisadas as notas explicativas das 113 empresas não financeiras listadas no segmento Novo Mercado da BM\&FBovespa, referentes ao exercício social de 2013. Trata-se de estudo descritivo com abordagem qualitativa e quantitativa. Preliminarmente, por meio de pesquisa documental, foram identificadas as informações de riscos de mercado divulgadas, utilizando-se check-list desenvolvido a partir de disposições constantes do Pronunciamento Técnico CPC 40 (R1) - Instrumentos Financeiros: Evidenciação, verificando-se que o número de divulgações de riscos de mercado das empresas é inferior a 50\% do requerido pelo normativo contábil. Em seguida, foram realizadas análises estatísticas de correlação e regressão linear com o intuito de verificar os fatores das empresas associados à divulgação de seus riscos de mercado. Com base nos resultados obtidos, verificou-se que os fatores "nível de risco”, "tamanho” e “setor de atuação” são significativos para explicar o cumprimento das empresas não financeiras às exigências definidas no CPC 40 (R1). Além disso, os resultados fornecem suporte para a aceitação da hipótese, mostrando que empresas com maior nível de risco divulgam mais informações sobre risco de mercado.

Palavras-chave: Divulgação. Informações. Risco de mercado. CPC 40 (R1). Empresas não financeiras.

\section{Abstract}

This study aimed to identify the determinants of the level of observance by non-financial companies to the disclosure requirements set out in CPC 40 (R1). That sense, it was analyzed the explanatory notes to the 113 non-financial companies listed on the New Market segment of the BM\&FBovespa relative the social year 2013. It is a descriptive study with qualitative and quantitative approach. Preliminarily, through documentary research, the disclosure of market risk information were identified, using check-list developed from the provisions of CPC 40 (R1) - Financial Instruments: Disclosure, verifying that the number of market risk disclosures in the company is less than $50 \%$ of required by regulatory accounting. Then, statistical analyzes were performed correlation and linear regression in order to identify factors associated companies to the disclosure of their market risks. Based on the results obtained, it was found that the factors "level of risk", "size" and "Sector of activity" this are significant to explain the performance of non-financial firms with the requirements set out in CPC 40 (R1). Furthermore, the results provide support for acceptance of the hypothesis by showing that companies with the highest risk level disclosure more information about market risk.

Keywords: Disclosure. Information. Market risk. CPC 40 (R1). Non-financial companies. 


\section{INTRODUÇÃO}

O risco é um elemento inevitável de qualquer empreendimento. No entanto, as crises financeiras do mercado e os escândalos financeiros ocorridos na última década obrigaram as empresas a se preocuparem mais com os investidores, fazendo com que a gestão de riscos e sua divulgação ganhassem maior relevância no meio empresarial (GRAÇA, 2012).

Além disso, a expansão no ambiente de negócios, a abertura de capital e a ampliação dos mercados aumentaram a importância da divulgação de risco corporativo, especialmente por empresas não financeiras. Conforme Elzahar e Hussainey (2012), as empresas tentam satisfazer às necessidades dos usuários da informação contábil por meio da divulgação de mais informações sobre os diferentes riscos a serem enfrentados e a sustentabilidade de suas operações. Essas informações ajudam os usuários a avaliarem o risco futuro, o que é essencial para otimizar suas operações mantendo carteiras bem diversificadas (ELZAHAR; HUSSAINEY, 2012).

A gestão de risco tornou-se consideravelmente mais importante nos últimos anos e reflete uma parte essencial da boa governança corporativa (HUNZIKER, 2013). Uma das particularidades do debate sobre riscos diz respeito à comunicação adequada das informações de risco pelas empresas aos seus stakeholders (LINSLEY; SHRIVES, 2006). Conforme Shinzato (2011), os prejuízos causados em companhias abertas pela crise financeira internacional de 2008 forçaram o aperfeiçoamento do processo de evidenciação do risco de mercado de forma que estas passaram a divulgar informações de risco não apenas de forma voluntária, mas também por imposição de órgãos reguladores.

Essa demanda sobre a divulgação relacionada com o risco acabou se refletindo nas International Financial Reporting Standards (IFRS). De acordo com a IFRS 7, empresas não financeiras precisam divulgar informações completas sobre os seus instrumentos financeiros e os riscos relacionados a esses instrumentos. No Brasil, a sujeição das empresas à divulgação do risco ocorre a partir de agosto de 2012, com a publicação do Pronunciamento Técnico CPC 40 (R1) - Instrumentos Financeiros: Evidenciação. O normativo, que se refere à evidenciação de instrumentos financeiros, requer para as companhias de capital aberto a divulgação de informações de caráter quantitativo e qualitativo sobre a exposição aos riscos decorrentes de instrumentos financeiros e como eles têm sido administrados, "incluindo divulgações mínimas específicas sobre risco de crédito, risco de liquidez e risco de mercado.” (COMITÊ DE PRONUNCIAMENTOS CONTÁBEIS, 2012, p. 3).

A preocupação do CPC 40 (R1), conforme explicitado em sua introdução, diz respeito a uma maior transparência em relação aos riscos de instrumentos financei- 
ros, permitindo que os usuários façam julgamentos mais balizados sobre o risco e o retorno (COMITÊ DE PRONUNCIAMENTOS CONTÁBEIS, 2012). Não obstante, conforme Welker (1995), apesar de serem dedicados consideráveis recursos para a criação e implementação de regulamentos destinados à melhoria das práticas de divulgação, as empresas ainda têm discricionariedade para determinar a oportunidade, âmbito, conteúdo e forma de divulgação de informações aos usuários externos, de modo que a regulação contábil não envolve apenas as leis, normas e pronunciamentos contábeis, mas, também, as exigências de cumprimento dessa legislação.

Desse modo, assumindo-se que as práticas de disclosure das empresas também dependem do enforcement dos órgãos reguladores (MURCIA, 2009) e que, no Brasil não há uma adequada infraestrutura de enforcement contábil (COSENZA; LAURENCEL, 2012), formulou-se a seguinte questão de pesquisa: quais os fatores determinantes do nível de observância das empresas não financeiras às exigências de divulgação definidas no CPC 40 (R1)?

Assim, neste estudo teve-se como objetivo identificar os fatores determinantes do nível de observância das empresas não financeiras às exigências de divulgação definidas no CPC 40 (R1). Fundamentando-se na perspectiva do disclosure obrigatório, parte-se da hipótese geral de que há associação positiva entre o nível de informações divulgadas e o nível de risco das empresas.

Visando alcançar o objetivo com este estudo, realizou-se análise documental das notas explicativas das demonstrações financeiras das empresas da amostra referentes ao exercício de 2013, com base em check-list elaborado a partir dos dispositivos contidos no CPC 40 (R1). Em seguida os dados foram analisados por meio das técnicas estatísticas de correlação e regressão linear.

O estudo se justifica em razão de a divulgação obrigatória de risco por empresas não financeiras ser uma prática contábil recente no País, carente de investigação, ampliando os achados de estudos similares sobre o tema no contexto brasileiro e contribuindo com evidências empíricas acerca do nível de divulgação de informações de risco de mercado por empresas não financeiras, em atendimento ao CPC 40 (R1).

\section{REFERENCIAL TEÓRICO}

\subsection{DIVULGAÇÃO DE RISCO DE MERCADO}

De acordo com Martins et al. (2013), é dever da administração garantir que acionistas e outras partes interessadas tenham acesso a divulgações de qualidade so- 
bre o estado atual e esperado dos negócios para que estes tenham uma melhor compreensão dos riscos a que estão expostos. Nesse contexto, a contabilidade desempenha papel essencial na elaboração de relatórios financeiros da empresa que possibilitam uma melhor compreensão dos riscos inerentes aos negócios. Sob a ótica da gestão de riscos, a lógica da divulgação das informações contábeis consiste, segundo Murcia (2009), na redução do grau de incerteza dos investidores a respeito dos resultados futuros da empresa, aumentando a eficiência do mercado.

Com o intuito de incentivar a divulgação de informações privadas por parte das empresas, considerando um monitoramento mais efetivo dos atos dos gestores por parte dos demais stakeholders e a consequente redução da assimetria informacional existente no mercado de capitais, foram criados mecanismos externos e internos de divulgação entre os quais se inclui o disclosure obrigatório, o qual compreende a divulgação de informações exigidas pela legislação pertinente e pelos órgãos reguladores competentes (KIRCH; LIMA; TERRA, 2012).

Bushman, Piotroski e Smith (2004) interpretam o disclosure como um fator de transparência financeira, relacionado ao conceito de governança corporativa, por referir-se à disponibilização de informações que interessam aos agentes de mercado. Avelino (2013) ressalta que o conjunto total do disclosure de uma empresa contém informações tanto compulsórias, que atendem aos requisitos da legislação e dos organismos reguladores, quanto voluntárias, as quais não têm requisito de obrigatoriedade.

Em relação à divulgação de informações voluntárias, Miihkinen (2012) afirma que quando não é exigida a evidenciação, algumas empresas podem não as divulgarem voluntariamente. Dessa forma, torna-se necessária a regulação ou normatização para melhorar a qualidade das informações no que se refere a aspectos como uniformidade, identificação de impactos econômicos e impactos no desempenho empresarial que afetem as relações com os stakeholders.

A regulação pode ser entendida como um conjunto de restrições intencionais emanadas de uma entidade reguladora (Estado ou órgão regulador), relativas à determinada atividade de um sujeito (CARDOSO et al., 2010). De modo específico, conforme os autores, o sistema de regulação da contabilidade de um país é influenciado por diferentes agentes (órgãos do Estado, organizações profissionais, instituições vinculadas ao mercado financeiro e academia contábil) que interagem entre si e agem sobre o próprio sistema, podendo ainda interagir com os padrões internacionais de contabilidade.

Para Bushee e Leuz (2005), um papel potencial da divulgação obrigatória é servir como um dispositivo de compromisso, já que impele as empresas a revelarem 
informações em bons e maus momentos. Conforme Murcia (2009, p. 37), "Os defensores da regulação do disclosure argumentam que as empresas não estão dispostas a aumentar o nível de divulgação de suas informações, a não ser quando obrigadas.” Assim, o disclosure obrigatório contribui para a redução da assimetria informacional ao impor às empresas a obrigatoriedade de divulgar informações que estas não divulgariam voluntariamente (MURCIA, 2009). Entretanto, conforme esclarece Murcia (2009), para que o disclosure emanado das leis, normas e pronunciamentos contábeis seja efetivo é necessário que seja acompanhado de um enforcement eficiente por parte dos órgãos reguladores, de forma que sua adequação em termos de cumprimento dos normativos depende da efetividade das ações desses órgãos.

Um dos objetivos da regulação é a redução da assimetria informacional. Particularmente, no que se refere às informações de risco, Abraham e Shrives (2014) afirmam que muitas práticas de divulgação de risco geralmente se mostram como válidas, no entanto, elas não informam efetivamente aos usuários nenhum conteúdo útil, pois apesar de muitas vezes serem obrigatórias, essas informações podem ser compostas de conteúdos limitados e de pouca relação com risco, não sendo eficazes para o processo tomada de decisão. Dobler (2008) considera que o custo da divulgação do risco é um fator coerente com a oposição das empresas em elaboração de relatórios obrigatórios, visto que uma informação sobre risco também demanda custos para a empresa.

Com base nos aspectos descritos nesta seção, percebe-se que a regulação contábil é uma forma de garantir que as empresas divulguem informações, mesmo quando não possuam motivação discricionária para isso. Pinho, Parente e Coelho (2014, p. 2) observam que o CPC 40 (R1) "visa proporcionar maior transparência ao mercado e dispor meios para que os agentes do mercado realizem uma avaliação eficiente sobre os riscos a que as empresas de capital aberto estão expostas." No entanto, como "as normas contábeis envolvem uma inerente subjetividade” (MURCIA, 2009, p. 43), a qualidade do disclosure será influenciada por outros fatores, além das prescrições normativas e legais, como características operacionais das empresas e estruturas de governança.

As informações prestadas pelas empresas ao público de interesse têm uma grande importância na tomada de decisão do usuário externo da contabilidade. No decorrer de suas atividades normais as empresas acabam sempre em situações de envolvimento com riscos. Qualquer suspeita de existência de risco pode influenciar o investimento de algum acionista, visto que essa informação deve ser prestada pelas empresas no sentido de informar ao investidor sobre a situação de risco em que a empresa está inserida. A partir desse contexto, surge a relevância da divulgação re- 
lacionada a essa matéria, como fator importante na decisão do público de interesse (MARTINS; PAULO, 2013). Assim, as decisões dos investidores são influenciadas pelas informações prestadas pelas empresas; quando se trata de evidenciação relacionada a risco, eles avaliam o retorno esperado e o risco, de modo que, quando detém esse tipo de informação, o usuário toma decisões com mais convicção (CABEDO; TIRADO, 2003).

Putri e Arofah (2013) indicam que uma informação completa sobre risco da empresa pode afetar a confiança dos participantes do mercado, no sentido de induzir a uma visão de maior credibilidade da empresa que divulga informações completas sobre risco, gerando confiança para os possíveis investidores. Na visão de Othman e Ameer (2009), os stakeholders necessitam de maior transparência nos relatórios financeiros com informações relevantes sobre risco de mercado para a tomada de decisão.

Fernandes (2012) considera a gestão de riscos como fundamental para as empresas, visto que estas estão expostas a vários tipos de risco como o risco de crédito, de mercado e operacional, e com isso a gestão desses riscos passa a ser algo importante em seus processos de desenvolvimento e geração de riquezas.

Conforme Linsley e Shrives (2006), o risco refere-se a qualquer oportunidade ou perspectiva, ou a qualquer perigo, dano, ameaça ou exposição que possa causar ou vir a causar impacto sobre a empresa. Esses autores consideram que há disclosure de risco quando o usuário é informado acerca de qualquer desses fatores que podem afetar o futuro da empresa e consequentemente o futuro do investimento.

Segundo Goulart (2003), o risco de mercado pode ser entendido como oscilações em variações econômicas ou financeiras de empresas. Nesse mesmo sentido, Guzzo et al. (2013) entendem que as empresas atuam em um mercado em que situações futuras são incertas e, com isso, estão expostas a riscos caracterizados por essa incerteza, podendo ser segregados em vários tipos de acordo que os causam, como risco de crédito, risco de liquidez, risco de mercado e outros. De acordo com Emm, Gay e Lin (2007), as empresas estão expostas a flutuações de preços decorrentes das variações de taxas de câmbio, taxas de juros e preços de commodities que são classificadas como risco de mercado. O risco de mercado, especificamente, está relacionado com perdas decorrentes de oscilações econômicas e financeiras, como flutuação das taxas de juros, preços de ações e outras causas que independem do controle da empresa.

O CPC 40 (R1), relativo à evidenciação de instrumentos financeiros, define risco de mercado como oscilações de valor justo ou fluxos de caixa de investimentos financeiros, quando essas oscilações estão ligadas a mudanças de preço de mercado, 
enfatizando que, além de outros riscos da empresa, devem ser divulgados também os riscos de mercado para que possibilitem aos usuários das informações contábeis maior transparência e consequentemente uma melhor avaliação da entidade em relação à exposição aos riscos (COMITÊ DE PRONUNCIAMENTOS CONTÁBEIS, 2012).

Ainda, de acordo com o CPC 40 (R1), "o risco de mercado compreende três tipos de risco: risco de moeda, risco de taxa de juro e outros riscos de preços.” (COMITÊ DE PRONUNCIAMENTOS CONTÁBEIS, 2012). Risco de moeda "é o risco de o valor justo ou os fluxos de caixa futuros de instrumento financeiro oscilarem devido a mudanças nas taxas de câmbio de moeda estrangeira.” (COMITÊ DE PRONUNCIAMENTOS CONTÁBEIS, 2012). Por sua vez, o risco de taxa de juros "é o risco de o valor justo ou os fluxos de caixa futuros de instrumento financeiro oscilarem devido a mudanças nas taxas de juro de mercado." (COMITÊ DE PRONUNCIAMENTOS CONTÁBEIS, 2012). Os outros riscos de preços são definidos pelo referido CPC como

\begin{abstract}
os riscos de o valor justo ou os fluxos de caixa futuros de instrumento financeiro oscilarem como resultado de alterações nos preços de mercado (que não são as que decorrem do risco de taxa de juros ou riscos cambiais), quer sejam essas alterações por fatores específicos do instrumento financeiro, ou fatores que afetam todos os instrumentos financeiros semelhantes negociados no mercado. (COMITÊ DE PRONUNCIAMENTOS CONTÁBEIS, 2012, p. 18).
\end{abstract}

Na concepção de Hunziker (2013), o risco de mercado é a principal categoria de risco financeiro a ser divulgado. Isso porque, segundo Othman e Ameer (2009), na ausência de informações sobre risco, os investidores são incapazes de identificar todos os fatores que afetam as condições financeiras da empresa, o que confere uma maior importância à evidenciação desse tipo de risco.

Segundo Blankley, Lamb e Schroeder (2002), a empresa deve divulgar informações quantitativas sobre risco de mercado e, além disso, informar as principais exposições ao risco, como administra e gerencia esses riscos, e as limitações que impedem de evidenciar completamente as demais informações relevantes. Isso leva a um cenário de maior controle por parte dos stakeholders, proporcionando maior segurança e conviç̧ão nos possíveis investimentos a serem realizados de acordo com informações mais precisas prestadas pelas empresas. 
De acordo com o CPC 40 (R1), a entidade deve divulgar informações qualitativas e quantitativas relativas à exposição a riscos decorrentes de instrumentos financeiros, "incluindo divulgações mínimas específicas sobre risco de crédito, risco de liquidez e risco de mercado.” (COMITÊ DE PRONUNCIMENTOS CONTÁBEIS, 2012, p. 3). No que se refere às informações qualitativas, o normativo define que, para cada tipo de risco decorrente de instrumentos financeiros, a entidade deve divulgar: a exposição ao risco e como ele surge; seus objetivos, políticas e processos para gerenciar os riscos e os métodos utilizados para mensurar o risco; e quaisquer alterações nesses elementos em relação ao período anterior.

Quanto às informações quantitativas, especificamente em relação ao risco de mercado, de acordo com o CPC 40 (R1), a entidade deve divulgar "uma análise de sensibilidade para cada tipo de risco de mercado aos quais a entidade está exposta ao fim do período contábil” (COMITÊ DE PRONUNCIAMENTOS CONTÁBEIS, 2012), a menos que ela elabore "uma análise de sensibilidade tal como a do valor em risco (value-at-risk) e o utilizar para administrar riscos financeiros.” (COMITÊ DE PRONUNCIAMENTOS CONTÁBEIS, 2012, p. 15).

A análise de sensibilidade deverá mostrar como o resultado e o patrimônio líquido da entidade seriam afetados pelas mudanças no risco de mercado (COMITÊ DE PRONUNCIAMENTOS CONTÁBEIS, 2012). Ainda de acordo com o CPC 40 (R1), a entidade deve divulgar "os métodos e os pressupostos utilizados na elaboração da análise de sensibilidade”, bem como a ocorrência de alterações nos métodos e pressupostos utilizados na análise, em relação ao período anterior, e as razões para essas alterações (COMITÊ DE PRONUNCIAMENTOS CONTÁBEIS, 2012, p. 15).

O valor em risco reflete interdependências entre riscos variáveis, como riscos de taxas de juros e riscos de taxa de câmbio (COMITÊ DE PRONUNCIAMENTOS CONTÁBEIS, 2012). De acordo com o CPC 40 (R1), caso a entidade faça uso do valor em risco, esta deverá divulgar também “uma explicação do método utilizado na elaboração de tal análise de sensibilidade e dos principais parâmetros e pressupostos subjacentes aos dados fornecidos” (COMITÊ DE PRONUNCIAMENTOS CONTÁBEIS, 2012) e, ainda, “uma explicação do objetivo do método utilizado e das limitações que podem resultar do fato da informação não refletir completamente o justo valor dos ativos e dos passivos envolvidos.” (COMITÊ DE PRONUNCIAMENTOS CONTÁBEIS, 2012, p. 15).

O CPC 40 (R1) refere-se, ainda, a “outras divulgações de risco de mercado”, relatando que, quando as análises de sensibilidade anteriormente explicitadas "não forem representativas do risco inerente de instrumento financeiro, a entidade deve di- 
vulgar esse fato e a razão pela qual considera que as análises de sensibilidade não são representativas.” (COMITÊ DE PRONUNCIAMENTOS CONTÁBEIS, 2012, p. 15).

\subsection{ESTUDOS EMPÍRICOS}

No Brasil, poucos estudos buscaram analisar o disclosure dos riscos incorridos pelas empresas. Dantas et al. (2010) realizaram pesquisa no sentido de avaliar o grau de transparência do risco de crédito praticado pelas instituições financeiras brasileiras, tomando como base o Acordo de Basileia II. Entre outros aspectos, os autores constataram que o porte das instituições, o nível de rentabilidade, o índice de imobilização e a listagem das ações na Bovespa são elementos explicativos do grau de evidenciação do risco de crédito pelas instituições financeiras da amostra.

Beuren, Dallabona e Dani (2011) analisaram o conteúdo do disclosure das informações sobre gestão de riscos e controle interno de empresas listadas na BM\&FBovespa em suas notas explicativas. Os resultados da pesquisa mostram que risco de mercado, risco de crédito e relatório do risco financeiro, nessa ordem, foram, entre outros itens, os mais divulgados pelas empresas analisadas.

Pinho, Parente e Coelho (2014) avaliaram a relação entre nível de disclosure obrigatório dos riscos decorrentes dos instrumentos financeiros e nível de risco das empresas, sob a hipótese de que quanto maior tal disclosure, menores riscos adviriam para investidores. Não foi constatada significância estatística para a existência de associação entre o nível de disclosure e as medidas de risco escolhidas.

No que se refere ao disclosure de risco de mercado, especificamente, mediante busca por palavras-chave na Web (Google acadêmico, Biblioteca de Teses da USP, Periódicos CAPES), não foram encontrados estudos empíricos relacionados à divulgação do risco de mercado por empresas não financeiras à luz do CPC 40 (R1), equivalente brasileiro da IFRS 7. No âmbito internacional, diversos estudos analisaram a divulgação de riscos pelas empresas.

Estudo realizado por Hodder et al. (2001) para verificar o disclosure de risco e suas implicações na tomada de decisão do usuário constatou que evidenciações de informações quantitativas e mais detalhadas sobre riscos podem mitigar os efeitos do receio do investidor, trazendo maior confiança e aumentando a credibilidade da empresa.

Blankley, Lamb e Schroeder (2002) analisaram a divulgação de riscos de mercado de 30 empresas que compõem o índice Dow 30 de acordo com normas emitidas pela SEC, as quais requerem divulgações qualitativas e quantitativas por parte das 
empresas. Os resultados apontaram que a análise de sensibilidade foi o método mais utilizado pela maioria das empresas para relatar os seus riscos de mercado, visto que, no geral, as informações divulgadas foram pouco detalhadas, não havendo evidenciação abrangente sobre como a empresa gerencia os riscos de mercado, caracterizando baixa qualidade na evidenciação.

Em um estudo sobre o disclosure de informações sobre risco de mercado, Abdelghany (2005) utilizou uma amostra de 323 empresas para verificar a existência de relação entre risco de mercado e medidas contábeis de risco, tendo encontrado associação significativa entre risco de mercado como variável beta e quatro variáveis descritas: o ativo total, o índice de liquidez corrente, a variável de crescimento dos lucros e a distribuição de dividendos. $\mathrm{O}$ autor conclui que as empresas com maior proporção de ativo circulante sobre o passivo circulante tendem a ter maior grau de risco de mercado.

Linsley e Shrives (2006) analisaram as divulgações de risco em uma amostra de 79 relatórios anuais de empresas do Reino Unido, por meio de análise de conteúdo. Eles verificaram haver uma associação significativa entre o número de divulgações de risco e o tamanho da empresa, bem como entre o número de divulgações de risco e o nível de risco ambiental, não tendo encontrado, entretanto, nenhuma associação entre o número de divulgações de risco e outras medidas, como índice de alavancagem financeira e cobertura de ativos.

Abraham e Cox (2007) realizaram um estudo sobre informações de risco nos relatórios anuais de empresas pertencentes ao índice FTSE 100, que compreende as 100 maiores companhias do Reino Unido em relação à capitalização de mercado. Foi constatado que a comunicação de riscos nas empresas é negativamente relacionada com a participação acionária de longo prazo das instituições. Os autores entendem que os investidores que aplicam em ações por um longo prazo tendem a investir em empresas com menor divulgação de informações sobre riscos, caracterizando os acionistas de longo prazo como investidores que não pressionam a empresa para uma maior evidenciação, o que justifica essa relação.

Othman e Ameer (2009) estudaram a divulgação de risco em empresas da Malásia e identificaram o cumprimento da norma de divulgação de risco de mercado, no entanto concluíram que faltam informações na divulgação para que estas tenham maior utilidade, implicando a necessidade de maior transparência na divulgação para que as expectativas dos stakeholders possam ser atendidas.

Estudo com uma amostra de 72 empresas do Reino Unido, realizado por Elzahar e Hussainey (2012) sobre a divulgação de risco mostrou que grandes empresas 
divulgam mais informações de risco, que a atividade da empresa está positivamente associada com níveis de divulgação e que o impacto de outras características específicas da empresa como liquidez e alavancagem financeira é estatisticamente insignificante.

Em um estudo com uma amostra de 99 empresas da Finlândia, Miihkinen (2012) examinou o impacto do padrão IFRS na divulgação de riscos de empresas em relação à melhoria da qualidade de informações e encontrou resultados que indicam melhoria da qualidade da divulgação de risco em decorrência dos padrões IFRS, mostrando que a adoção do padrão impacta as informações sobre risco, além de apresentar evidências de que os impactos trazidos pelas IFRS são maiores em empresas com menor rentabilidade. Savvides e Savvidou (2012) realizaram um estudo para investigar as práticas de divulgação de risco de mercado de 30 bancos de vários países diferentes e encontraram relação positiva e estatisticamente significante entre o tamanho da empresa e o nível de divulgação.

Estudo realizado por Hunziker (2013) explorou as divulgações de risco de mercado em uma amostra de 116 relatórios anuais de empresas não financeiras suíças listadas, por meio de análise de conteúdo e análise de correlação. O autor foi um dos primeiros a testar a relação entre divulgações de risco de mercado e desempenho da empresa. O estudo conclui que a análise de sensibilidade é o método predominante na divulgação dos riscos de mercado e também verificou que associações significativas são encontradas entre o número/quantidade de divulgações de risco de mercado e o tamanho da empresa. Da mesma forma, uma associação significativa foi encontrada entre o número/quantidade de divulgações de risco e o risco da empresa aproximado pelo quociente de endividamento. No entanto, não foi encontrada nenhuma associação entre o número/quantidade de divulgações de risco e o desempenho da empresa.

Investigando o disclosure de risco de empresas não financeiras do Reino Unido Elshandidy, Fraser e Hussainey (2013) encontraram que empresas maiores, com maior distribuição de dividendos e maior independência do conselho estão mais propensas a divulgarem informações tanto voluntárias quanto obrigatórias sobre riscos. A respeito de divulgação de risco de empresas financeiras, Hassan (2014) investigou os relatórios anuais de 23 empresas financeiras dos Emirados Árabes Unidos e concluiu que essas empresas utilizam as práticas de divulgação de risco para conseguir legitimidade perante a sociedade.

No estudo de Abraham e Shrives (2014), com o propósito de avaliar a qualidade da evidenciação de riscos de empresas do índice FTSE 100 do setor de produção e processamento de alimentos, com informações dos relatórios anuais dos anos 2002 
a 2007, os resultados indicaram que as divulgações de risco tendem a ter informações que não condizem com os riscos que ocorrem de fato nas empresas, visto que os gestores divulgam apenas informações sobre boas práticas de gestão de riscos, tornando as práticas de divulgação restritas e sem utilidade para o usuário.

Al-Shammari (2014) investigou a associação entre a divulgação do risco de mercado e algumas variáveis com uma amostra de 109 empresas e encontrou resultados indicando associação positiva entre a divulgação do risco de mercado e o tamanho da empresa, liquidez e tipo de auditoria da empresa, concluindo, ainda, que os resultados corroboram a teoria da agência e a teoria da sinalização.

\section{METODOLOGIA}

O normativo contábil CPC 40 (R1) impõe que a entidade divulgue nas suas demonstrações contábeis informações que permitam que os usuários avaliem a natureza e a extensão dos riscos a que a entidade está exposta, incluindo-se o risco de mercado. Conforme Jensen e Meckling (1976), empresas com exposições de alto risco induzem custos de agência mais elevados e, desse modo, precisam divulgar mais informações sobre o risco para os credores. Tomando por base os referenciais teóricos apresentados e as conclusões de Hunziker (2013), formulou-se a hipótese de que há associação positiva entre o nível de divulgações de risco de mercado e o nível de risco das empresas.

Para a análise da hipótese delineada e a verificação das características das empresas que influenciam o nível de evidenciação de risco de mercado foi realizado estudo descritivo, quantitativo e qualitativo, com abordagem quantitativa na análise dos dados, por meio de pesquisa documental, seguindo procedimentos adotados por Linsley e Shrives (2006) e outros trabalhos sobre divulgação de riscos (DANTAS et al., 2010; HUNZIKER, 2013; ELZAHAR; HUSSAINEY, 2012), compreendendo duas etapas: análise de conteúdo e procedimentos estatísticos de análise de coeficientes de correlação e regressão linear.

A condução da análise de conteúdo, segundo Bardin (1977), envolve várias etapas organizadas em três fases: pré-análise; exploração do material; tratamento dos resultados, inferência e interpretação. A primeira fase compreende a sistematização das ideias iniciais e o estabelecimento dos indicadores para a interpretação das informações coletadas. A fase de exploração do material envolve as operações de codificação e enumeração em razão de regras previamente formuladas. Na terceira fase os dados devem ser tratados de forma a serem significativos e válidos. Na codificação, 
efetuada seguindo regras precisas, os dados brutos do texto são transformados, por recorte, agregação e enumeração, permitindo uma representação do conteúdo, capaz de esclarecer as características do texto e que podem servir de índices (BARDIN, 1977).

Considerando-se os objetivos com este estudo, na análise de conteúdo teve-se como objeto as notas explicativas dos demonstrativos financeiros das empresas da amostra, visando à obtenção de dados quantitativos significativos para a identificação dos fatores determinantes do nível de observância das empresas não financeiras às exigências de divulgação definidas no CPC 40 (R1), sem a pretensão de usar essa técnica para avaliar a qualidade das divulgações de risco de mercado. Desse modo, para a execução da análise de conteúdo, foi aplicado check-list composto de 21 itens representativos de exigências de divulgação definidas no CPC 40 (R1) e seu Apêndice B, conforme Quadro 1. Dos 21 itens relacionados, 14 apresentam exigências de caráter qualitativo e os outros sete se referem a informações de caráter quantitativo.

Quadro 1 - Disclosure de risco de mercado em conformidade com o CPC 40 (R1)

\begin{tabular}{|c|c|}
\hline \multirow{14}{*}{ Itens qualitativos } & Declaração de exposição ao risco de moeda \\
\hline & Declaração de exposição ao risco de taxa de juros \\
\hline & Declaração de exposição a outros riscos de preços \\
\hline & Condições de mercado que dão origem ao risco de moeda \\
\hline & Condições de mercado que dão origem ao risco de taxa de juros \\
\hline & Condições de mercado que dão origem a outros riscos de preços \\
\hline & Objetivos da administração para gerenciar os riscos \\
\hline & Políticas e processos da administração para gerenciar os riscos \\
\hline & Métodos utilizados para mensurar o risco de moeda \\
\hline & Métodos utilizados para mensurar o risco de taxa de juros \\
\hline & Métodos utilizados para mensurar outros riscos de preços \\
\hline & Alterações da exposição ao risco em relação ao período anterior \\
\hline & Alteração dos objetivos da gestão de riscos em relação ao período anterior \\
\hline & Alteração das políticas de gestão de riscos em relação ao período anterior \\
\hline \multirow{7}{*}{$\begin{array}{l}\text { Itens quantita- } \\
\text { tivos }\end{array}$} & Análise de sensibilidade para risco de moeda \\
\hline & Análise de sensibilidade para risco de taxa de juros \\
\hline & Análise de sensibilidade para outros riscos de preços \\
\hline & $\begin{array}{l}\text { Métodos e pressupostos utilizados na elaboração da análise de sensibilidade para } \\
\text { risco de moeda }\end{array}$ \\
\hline & $\begin{array}{l}\text { Métodos e pressupostos utilizados na elaboração da análise de sensibilidade para } \\
\text { risco de taxa de juros }\end{array}$ \\
\hline & $\begin{array}{l}\text { Métodos e pressupostos utilizados na elaboração da análise de sensibilidade para } \\
\text { outros riscos de preços }\end{array}$ \\
\hline & Análise de sensibilidade como valor em risco (value-at-risk) \\
\hline
\end{tabular}

Fonte: os autores. 
A coleta de dados foi realizada com o auxílio de fichas padronizadas, elaboradas por meio de planilhas eletrônicas do Microsoft Excel para classificar os itens evidenciados pelas empresas sobre os riscos de mercado. Na tabulação dos dados, consideraram-se as informações dicotômicas, sendo atribuída a pontuação “1” para as empresas que evidenciaram informações relativas ao item analisado e “ 0 ” caso não especificassem informações sobre tais itens.

Na coleta dos dados, se a empresa declarou, por exemplo, que não está exposta a risco de moeda, considerou-se que ela evidenciou informação relativa à exposição ao risco de moeda e que não se aplicam os demais itens referentes a esse tipo de risco, quais sejam: condições de mercado que dão origem ao risco de moeda; métodos utilizados para mensurar o risco de moeda; análise de sensibilidade para risco de moeda; e métodos e pressupostos utilizados na elaboração da análise de sensibilidade para risco de moeda.

Os dados referentes à divulgação de riscos de mercado pelas empresas foram obtidos por meio de análise documental das notas explicativas, disponibilizadas no website da BM\&FBovespa, coletados no período de 27 de outubro a 05 de novembro de 2014. Compõem a amostra 113 empresas não financeiras listadas no segmento de governança corporativa Novo Mercado da BM\&FBovespa. A opção pelas empresas listadas no segmento Novo Mercado da BM\&FBovespa decorre do fato de que a listagem nesse segmento especial implica a adoção de medidas de governança corporativa adicionais às exigidas pela legislação brasileira, que ampliam os direitos dos acionistas, além da adoção de uma política de divulgação de informações mais transparente e abrangente.

Após a obtenção dos quantitativos de informações de risco de mercado, a partir da análise de conteúdo, os dados foram submetidos a tratamento estatístico, compreendendo análise de estatística descritiva, análise de correlação e regressão linear múltipla. Considerando-se a hipótese anteriormente formulada, utilizou-se como variável dependente o Nível de divulgação do risco de mercado (NDIV), obtido pela razão entre o número de informações divulgadas e o número de itens aplicáveis a cada empresa, e como variável explicativa, o Nível de risco da empresa (RISK), considerando-se o trabalho seminal de Modigliani e Miller (1958), os quais sugerem que um maior grau de alavancagem (debt-equity ratio) da empresa está associado a um maior retorno de seus ativos e, consequentemente, a um maior risco.

Foram utilizadas também as variáveis de controle Tamanho (DIM), Desempenho (DES) e Setor (SET). As proxies e fórmulas de cálculo para as variáveis independentes estão definidas no Quadro 2. 
Assim, em razão do objetivo do estudo, construiu-se o seguinte modelo de análise, tendo nível de divulgação de risco de mercado como variável dependente:

$$
N D I V=\beta_{0}+\beta_{1} R I S K+\beta_{2} D I M+\beta_{3} D E S+\beta_{4} S E T+\varepsilon
$$

Quadro 2 - Definição e mensuração das variáveis

\begin{tabular}{|c|c|c|c|}
\hline $\begin{array}{l}\text { Variável indepen- } \\
\text { dente }\end{array}$ & Proxies utilizadas & Fórmula de cálculo & Fundamentação \\
\hline $\begin{array}{l}\text { Risco da empresa } \\
\text { (RISK) }\end{array}$ & Debt-Equity Ratio & $\begin{array}{l}\text { Capitais de terceiros/Capitais } \\
\text { próprios }\end{array}$ & $\begin{array}{l}\text { Modigliani e Miller } \\
\text { (1958) e Silva (2012) }\end{array}$ \\
\hline Tamanho (DIM) & Dimensão & $\begin{array}{l}\text { Logaritmo natural do ativo } \\
\text { total }\end{array}$ & $\begin{array}{l}\text { Soares e Galdi (2011) } \\
\text { e Silva (2012) }\end{array}$ \\
\hline Desempenho (DES) & $\begin{array}{l}\text { Retorno sobre os Ativos } \\
\text { (ROA) }\end{array}$ & Lucro líquido/ativo total & $\begin{array}{l}\text { Hunziker (2013) e } \\
\text { Silva (2012) }\end{array}$ \\
\hline Setor (SET) & $\begin{array}{l}\text { Uma variável Dummy } \\
\text { para cada setor de } \\
\text { atuação da empresa de } \\
\text { acordo com a BM\&- } \\
\text { FBovespa. Atribuindo- } \\
\text {-se “1” quando a empre- } \\
\text { sa pertencer ao setor em } \\
\text { questão e “0” quando a } \\
\text { empresa pertencer aos } \\
\text { outros setores relacio- } \\
\text { nados. }\end{array}$ & $\begin{array}{l}\text { Construção e transporte = } 1 \\
\text { (Sim), } 0 \text { (Não); Tecnologia } \\
\text { da informação = } 1 \text { (Sim), } 0 \\
\text { (Não); Consumo cíclico = } 1 \\
\text { (Sim), } 0 \text { (Não); Consumo não } \\
\text { cíclico = } 1 \text { (Sim), } 0 \text { (Não); } \\
\text { Materiais básicos = } 1 \text { (Sim), } 0 \\
\text { (Não); Utilidade pública = } 1 \\
\text { (Sim), } 0 \text { (Não); } \\
\text { Bens industriais = } 1 \text { (Sim), } \\
0 \text { (Não); } \\
\text { Petróleo, gás e biocombustí- } \\
\text { veis = } 1 \text { (Sim), } 0 \text { (Não); } \\
\text { Telecomunicações = } 1 \text { (Sim), } \\
0 \text { (Não). }\end{array}$ & $\begin{array}{l}\text { Mapurunga, Ponte e } \\
\text { Holanda (2014) }\end{array}$ \\
\hline
\end{tabular}

Fonte: os autores.

Os dados para a aplicação da fórmula de cálculo das proxies foram obtidos a partir dos demonstrativos financeiros padronizados (demonstração do resultado e balanço patrimonial) das empresas da amostra, disponibilizados no website da BM\&FBovespa, referentes ao ano 2013. As análises estatísticas foram realizadas com o apoio do software IBM SPSS Statistic 21.

\section{ANÁLISE DOS RESULTADOS}

Os resultados do estudo apontaram, por meio da análise de conteúdo das notas explicativas, que a totalidade das empresas observadas (113 empresas) informou estar exposta a riscos de taxas de juros, 92\% declararam exposição ao risco de moeda ou de não sujeição a esse tipo de risco em razão da não realização de transações em outras moedas, e 27\% apresentaram declaração de exposição a outros riscos de pre- 
ços. Ressalte-se que os outros riscos de preços decorrem de instrumentos financeiros em razão de mudanças em preços de commodities ou preços de ações, não sendo aplicável a todas as empresas. No entanto, a grande maioria delas não apresenta declaração nesse sentido.

A proporção das empresas que declararam exposição aos riscos de mercado e especificaram as origens desses riscos foi de $71 \%$ para o risco de moeda, $92 \%$ para o risco de taxa de juros e $81 \%$ para outros riscos de preços. Por sua vez, a proporção de empresas que especificaram seus objetivos, políticas e processos para gerenciar riscos se situa em cerca de 57\%. Por outro lado, os itens qualitativos relativos aos métodos utilizados para mensurar o risco apresentaram percentual de divulgação inferior a 3\%. Apenas uma empresa da amostra divulgou informação sobre alterações nos métodos e nos pressupostos utilizados em períodos anteriores. Isso pode ter ocorrido simplesmente porque não ocorreram alterações nesses métodos, embora nenhuma empresa tenha explicitado essa informação nas notas explicativas.

No que se refere aos itens quantitativos, verificou-se que, das empresas que declararam exposição aos riscos de mercado, 60\% apresentaram análise de sensibilidade para risco de moeda, 93\% para risco de taxa de juros e $42 \%$ para outros riscos de preço.

Por sua vez, a quase totalidade das empresas que realizou análise de sensibilidade declarou os métodos e pressupostos utilizados na elaboração dessa análise, com exceção de apenas quatro empresas que não apresentaram tal declaração para os riscos de taxas de juros, corroborando os trabalhos de Blankley, Lamb e Schrolder (2002) e Hunziker (2013), os quais apontaram a análise de sensibilidade como método predominante para a divulgação do risco de mercado. Apenas quatro empresas apresentaram análise de sensibilidade value-at-risk; três delas também apresentaram análise de sensibilidade para cada tipo de risco de mercado aplicável.

A Tabela 1 mostra a estatística descritiva das variáveis relacionadas ao nível de divulgação de informações sobre risco de mercado pelas empresas da amostra. 
Tabela 1 - Estatística descritiva e variáveis

\begin{tabular}{|c|c|c|c|c|c|c|}
\hline Variável & N. de observações & Mínimo & Máximo & Média & $\begin{array}{l}\text { Desvio } \\
\text { padrão }\end{array}$ & Variância \\
\hline NDIV & 114 & 0,0952 & 0,7143 & 0,4293 & 0,1285 & 0,0165 \\
\hline DIM & 114 & 11,5889 & 17,6575 & 14,6921 & 1,1239 & 1,2632 \\
\hline RISK & 114 & $-2,5579$ & 15,1784 & 1,2794 & 2,1204 & 4,4960 \\
\hline DES & 114 & $-2,4696$ & 0,7787 & 0,0053 & 0,3259 & 0,1062 \\
\hline \multicolumn{7}{|l|}{ Setor: } \\
\hline $\begin{array}{l}\text { Construção e } \\
\text { transporte }\end{array}$ & 114 & 0 & 1 & 0,2743 & 0,4482 & 0,2009 \\
\hline $\begin{array}{l}\text { Tecnologia da } \\
\text { informação }\end{array}$ & 114 & 0 & 1 & 0,0354 & 0,1856 & 0,0345 \\
\hline $\begin{array}{l}\text { Consumo cí- } \\
\text { clico }\end{array}$ & 114 & 0 & 1 & 0,2035 & 0,4044 & 0,1636 \\
\hline $\begin{array}{l}\text { Consumo não } \\
\text { cíclico }\end{array}$ & 114 & 0 & 1 & 0,2035 & 0,4044 & 0,1636 \\
\hline $\begin{array}{l}\text { Materiais bá- } \\
\text { sicos }\end{array}$ & 114 & 0 & 1 & 0,0708 & 0,2576 & 0,0664 \\
\hline $\begin{array}{l}\text { Utilidade pú- } \\
\text { blica }\end{array}$ & 114 & 0 & 1 & 0,0885 & 0,2853 & 0,0814 \\
\hline Bens industriais & 114 & 0 & 1 & 0,0885 & 0,2853 & 0,0814 \\
\hline $\begin{array}{l}\text { Petróleo, gás } \\
\text { e biocombus- } \\
\text { tíveis }\end{array}$ & 114 & 0 & 1 & 0,0265 & 0,1615 & 0,0261 \\
\hline
\end{tabular}

A análise da estatística descritiva das variáveis mostra que o menor nível de divulgação pelas empresas foi 0,09, sendo caracterizado como um nível muito baixo de divulgação. Em relação ao nível máximo de divulgação, percebe-se que para a amostra em questão, a empresa que mais divulgou teve um nível acima de 0,7, caracterizando um alto nível, visto que foi bem próximo da divulgação total $(1,0)$ de acordo com o modelo utilizado para o cálculo desse nível. Desse modo, pode-se perceber que nenhuma empresa deixou de divulgar informações sobre risco de mercado. Em média, o número dessa divulgação foi 0,43 , mostrando que o nível de divulgação de risco de mercado pelas empresas da amostra é inferior à metade do exigido pelo CPC 40 (R1).

Em relação ao tamanho das empresas, determinado pelo logaritmo natural do ativo total, sua média foi maior que 14 com desvio padrão de 1,12, caracterizando certa homogeneidade, ao passo que a média de desempenho, medida pelo ROA, foi muito próxima a zero $(0,005)$, com desvio padrão de 0,32 , caracterizando homogeneidade razoável para os dados, ressaltando que a referida homogeneidade razoável foi possível em razão da exclusão de empresa altamente deficitária (outlier) da amostra. 
Quanto ao nível de risco da empresa (RISK), medido pelo quociente entre capitais de terceiros e capitais próprios, verificou-se um risco mínimo de -2,55 e máximo de 15,17, com média de 1,27 e desvio padrão de 2,12. O fato de o desvio padrão ter sido maior que a média significa que o nível de risco se mostra muito heterogêneo, com empresas apresentando alto nível de risco e outras, baixo nível.

Para avaliar o grau de relacionamento entre as variáveis definidas no estudo, foi realizada análise de correlação, conforme apresentado na Tabela 2.

Tabela 2 - Correlação entre as variáveis

\begin{tabular}{|c|c|c|c|c|}
\hline Variável & NDIV & DIM & RISK & DES \\
\hline NDIV & 1 & $0,242 * * *$ & 0,174 & $-0,044$ \\
\hline DIM & $0,242 * * *$ & 1 & 0,035 & $0,131 *$ \\
\hline RISK & 0,174 & 0,035 & 1 & $0,182 * *$ \\
\hline DES & $-0,044$ & $0,131^{*}$ & $0,182^{* *}$ & 1 \\
\hline
\end{tabular}

De acordo com a Tabela 2, o tamanho da empresa (DIM) está positivamente correlacionado com o nível de divulgação de risco de mercado (NDIV) ao nível de significância de $1 \%$, de forma que quanto maior a empresa, maior o nível de divulgação. A relação entre o desempenho (DES) e a divulgação não se mostrou significante. O desempenho associa-se positivamente com o tamanho da empresa, com relação significante ao nível de $10 \%$. O risco (RISK) é positivamente correlacionado com o desempenho com nível de significância de 5\%. Já as relações entre risco e divulgação de risco não se mostram significantes, assim como a relação entre o risco e o tamanho.

Com o intuito de identificar possíveis associações entre a variável dependente (NDIV) e as variáveis independentes, bem como indícios de multicolinearidade entre as variáveis independentes, foi aplicada a análise de regressão. 
Tabela 3 - Modelo de regressão

\begin{tabular}{|c|c|c|c|c|c|c|c|}
\hline \multirow{2}{*}{ Variável } & \multicolumn{2}{|c|}{$\begin{array}{c}\text { Coeficientes não } \\
\text { padronizados }\end{array}$} & \multirow{2}{*}{$\begin{array}{c}\begin{array}{c}\text { Coeficien- } \\
\text { te padro- } \\
\text { nizado }\end{array} \\
\text { Beta }\end{array}$} & \multirow{2}{*}{ Teste $\mathbf{t}$} & \multirow{2}{*}{$\begin{array}{l}\text { Signifi- } \\
\text { cância }\end{array}$} & \multicolumn{2}{|c|}{$\begin{array}{l}\text { Estatísticas de } \\
\text { colinearidade }\end{array}$} \\
\hline & B & $\begin{array}{l}\text { Modelo } \\
\text { padrão }\end{array}$ & & & & $\begin{array}{c}\text { Tole- } \\
\text { rância }\end{array}$ & VIF \\
\hline (Constante) & $-0,0042$ & 0,1725 & - & $-0,0245$ & 0,9805 & - & - \\
\hline DIM & 0,0267 & 0,0117 & 0,2337 & 2,2745 & 0,0250 & 0,7671 & 1,3037 \\
\hline RISK & 0,0105 & 0,0059 & 0,1734 & 1,7934 & 0,0759 & 0,8655 & 1,1553 \\
\hline DES & $-0,0274$ & 0,0436 & $-0,0696$ & $-0,6295$ & 0,5304 & 0,6627 & 1,5089 \\
\hline $\begin{array}{l}\text { Construção e } \\
\text { transporte }\end{array}$ & 0,0077 & 0,0664 & 0,0112 & 0,1162 & 0,9077 & 0,8789 & 1,1378 \\
\hline $\begin{array}{l}\text { Tecnologia da } \\
\text { informação }\end{array}$ & 0,0108 & 0,0354 & 0,0339 & 0,3045 & 0,7614 & 0,6534 & 1,5304 \\
\hline Consumo cíclico & 0,0706 & 0,0344 & 0,2221 & 2,0523 & 0,0427 & 0,6912 & 1,4468 \\
\hline $\begin{array}{l}\text { Consumo não } \\
\text { cíclico }\end{array}$ & 0,0583 & 0,0529 & 0,1169 & 1,1011 & 0,2735 & 0,7181 & 1,3926 \\
\hline Materiais básicos & $-0,0225$ & 0,0457 & $-0,0501$ & $-0,4930$ & 0,6231 & 0,7850 & 1,2739 \\
\hline Utilidade pública & 0,0989 & 0,0446 & 0,2196 & 2,2150 & 0,0290 & 0,8238 & 1,2139 \\
\hline Bens industriais & 0,0067 & 0,0827 & 0,0084 & 0,0807 & 0,9358 & 0,7485 & 1,3360 \\
\hline $\begin{array}{l}\text { Petróleo, gás e } \\
\text { biocombustíveis }\end{array}$ & $-0,0062$ & 0,1264 & $-0,0045$ & $-0,0489$ & 0,9611 & 0,9453 & 1,0579 \\
\hline $\mathrm{R}^{2}=0,182$ & & & & $F=0,031$ & $\mathrm{~N}$. & observ & es $=113$ \\
\hline
\end{tabular}

Fonte: os autores.

Nota: Variável dependente: NDIV.

A Tabela 3 mostra que o modelo é significante ao nível de 5\%, visto que o teste "F" foi menor que 0,05 . O R² indica o poder de explicação do modelo, em que apesar de as variáveis terem relações significantes, elas não conseguem explicar bem o nível de divulgação de risco de mercado, visto que além dessas variáveis, outras características da empresa podem afetar a divulgação. De acordo com o $\mathrm{R}^{2}$, as variáveis independentes do modelo explicam em média 18,2\% da divulgação do risco de mercado.

Em relação às estatísticas de colinearidade, vê-se que as relações entre as variáveis independentes (DIM, RISK e DES), inclusive as dummies, e a variável dependente (NDIV) não apresentaram problemas com multicolinearidade, visto que os valores Variance Inflation Factor (VIF) foram todos baixos.

A análise da regressão indica que o nível de divulgação de risco de mercado (NDIV) está associado com o nível de risco da empresa (RISK), com coeficiente positivo e significância de 10\%. Diante disso, empresas buscam adotar uma postura mais transparente no intuito de demonstrar que evidenciam suas informações negati- 
vas relacionadas a riscos, no sentido de fazer ciência dos riscos a que os investidores estão expostos, dando mais confiança e proporcionando maior segurança nas decisões de investimentos.

Do mesmo modo, verificou-se que o tamanho (DIM) da empresa impacta positivamente o nível de divulgação, pois a relação foi significante a 5\%, indicando que empresas maiores estão mais propensas a divulgarem mais informações sobre risco de mercado. Os resultados corroboram os estudos de Al-Shammari (2014), Elzahar e Hussainey (2012), Savvides e Savvidou (2012) e Linsley e Shrives (2006), que encontraram associação positiva entre a divulgação do risco e o tamanho da empresa.

Dessa maneira, as empresas de maior tamanho podem estar motivadas a terem um nível maior de divulgação de risco em razão de pressões sociais, visto que, por serem maiores, estão mais expostas às condições da mídia e precisam demonstrar que são mais transparentes tanto para questões relacionadas à manutenção de suas posições dentro do mercado quanto para adquirir maior confiança perante os stakeholders.

Já quanto ao desempenho (DES), não foi encontrada relação significativa entre desempenho e divulgação de risco de mercado, corroborando o estudo de Hunziker (2013), que também não encontrou nenhuma associação significativa entre o desempenho da empresa e o nível de divulgação de risco de mercado.

Em relação aos setores de atuação, conforme pode ser visto no modelo de regressão (Tabela 3), existe relação significativa com a divulgação de risco de mercado apenas entre os setores de utilidade pública (significância de 5\%) e consumo cíclico (significância de 10\%). Assim, pode-se inferir que empresas pertencentes aos setores de utilidade pública e de consumo cíclico tendem a apresentar maior nível de divulgação de informações de risco de mercado.

\section{CONSIDERAÇÕES FINAIS}

Neste estudo teve-se por objetivo identificar os fatores determinantes do nível de observância das empresas não financeiras listadas no segmento Novo Mercado da BM\&FBovespa ao CPC 40 (R1), no que se refere à divulgação do risco de mercado. Nessa perspectiva, foi realizado um estudo descritivo com abordagem quantitativa, por meio de pesquisa documental nas notas explicativas divulgadas pelas empresas, referentes ao ano 2013, mediante utilização de check-list composto de 21 itens pautados no CPC 40 (R1), para verificar a evidenciação de informações de riscos de mercado pelas empresas. 
Com base em dados obtidos por meio de análise de conteúdo, apurou-se que as empresas da amostra divulgam em média 48\% das informações requeridas pelo CPC 40 (R1), mostrando que o nível de divulgação de risco de mercado se apresenta inferior à metade do exigido pelo normativo contábil. Não obstante, observou-se que itens mais objetivos como declaração da exposição ao risco e como ele surge; indicação de objetivos, políticas e processos para gerenciar os riscos; e análise de sensibilidade para os riscos apresentaram altos níveis de divulgação, em contraste com itens relacionados à divulgação de métodos utilizados para gerenciar riscos, bem como à divulgação de alterações na exposição ao risco, nos objetivos e políticas de gerenciamento de riscos. Embora nenhuma das empresas analisadas tenha apresentado informação nesse sentido, acredita-se que mudanças como as descritas anteriormente não tenham ocorrido no âmbito das empresas analisadas, razão pela qual se justifica a ausência de informações desse tipo nas notas explicativas.

O estudo teve como ponto de partida a hipótese de que haveria uma associação positiva entre o nível de divulgação de risco de mercado e o nível de risco da empresa. Os resultados da pesquisa fornecem suporte para a aceitação da hipótese do estudo, mostrando que empresas com maior nível de risco divulgam mais informações sobre risco de mercado. Tal constatação corrobora o resultado encontrado por Hunziker (2013), o qual verificou que o nível de risco da empresa influencia positivamente a divulgação do risco de mercado, para uma amostra de empresas suíças.

O estudo verificou, também, a associação entre o nível de divulgação de risco de mercado e características das empresas como tamanho e setor de atividade. Nos resultados encontrados, como esperado, verificou-se associação positiva e significante quanto ao tamanho da empresa. Esse resultado é coerente com diversos estudos anteriores (LINSLEY; SHRIVES, 2006; DANTAS et al., 2010; ELZAHAR; HUSSAINEY, 2012; SAVVIDES; SAVVIDOU, 2012; HUNZIKER, 2013; ELSHANDIDY; FRASER; HUSSAINEY, 2013; AL-SHAMMARI, 2014), que mostram que empresas maiores divulgam mais informações de risco de mercado.

Quanto ao desempenho, foi encontrada relação com coeficiente negativo, porém sem significância estatística, não sendo possível inferir que empresas com maior desempenho divulgam menos informações sobre risco de mercado.

No que se refere aos setores de atividades, verificou-se associação significativa dos setores de consumo cíclico e de utilidade pública com o nível de divulgação de risco de mercado.

Com base nos resultados do estudo, conclui-se que características das empresas como nível de risco, tamanho da empresa e alguns setores de atividades são fa- 
tores significativos para explicar a observância das empresas não financeiras listadas no segmento Novo Mercado da BM\&FBovespa ao CPC 40 (R1), no que se refere à divulgação dos riscos de mercado.

Em decorrência do baixo poder explicativo das variáveis utilizadas no modelo, indicado pelo valor do $\mathrm{R}^{2}$, sugere-se para pesquisas futuras incluir outras variáveis para testar o nível de divulgação de risco de mercado. Além disso, outros estudos podem abordar a qualidade das informações divulgadas e ampliar as observações, incluindo as empresas dos demais níveis de governança corporativa e/ou do mercado tradicional da BM\&FBovespa, não consideradas no presente estudo.

\section{REFERÊNCIAS}

ABDELGHANY, K. E. Disclosure of market risk or accounting measures of risk: an empirical study. Managerial Auditing Journal, v. 20, i. 8, p. 867-875, 2005.

ABRAHAM, S.; COX, P. Analysing the determinants of narrative risk information in UK FTSE 100 annual reports. The British Accounting Review, v. 3, i. 39, p. 227-248, 2007.

ABRAHAM, S.; SHRIVES, P. J. Improving the relevance of risk factor disclosure in corporate annual reports. The British Accounting Review, v. 46, i. 1, p. 91-107, 2014.

AL-SHAMMARI, B. Kuwait corporate characteristics and level of risk disclosure: a content analysis approach. Journal of Contemporary Issues in Business Research, v. 3, i. 3, p. 128-153, 2014.

AMEER, R. Value-relevance of foreign-exchange and interest-rate derivatives disclosure the case of Malaysian firms. The Journal of Risk Finance, v. 10, i. 1, p. 78-90, 2009.

AVELINO, B. C. Características explicativas do nível de disclosure voluntário de municípios do estado de Minas Gerais: uma abordagem sob a ótica da teoria da divulgação. 2013. 176 p. Dissertação (Mestrado em Contabilidade e Controladoria)Universidade Federal de Minas Gerais, Belo Horizonte, 2013.

BARDIN, L. Análise de conteúdo. Lisboa: Edições 70, 1977. 
BEUREN, I. M.; DALLABONA, L. F.; DANI, A. C. Disclosure de informações sobre gestão de riscos e controle interno pelas empresas listadas na BM\&FBOVESPA. Revista de Economia e Administração, v. 10, n. 1, p. 44-65, jan./mar. 2011.

BLANKLEY, A.; LAMB, R.; SCHROEDER, R. The disclosure of information on market risk: evidence from the Dow 30. Managerial Auditing Journal, v. 17, i. 8, p. 438-45, 2002.

BUSHEE, B.; LEUZ, C. Economic consequences of SEC disclosure regulation: evidence from OTC bulletin board. Journal of Accounting and Economics, v. 39, p. 233-264, 2005.

BUSHMAN, R.; PIOTROSKI, J.; SMITH, A. What determines corporate transparency. Journal of Accounting Research, v. 42, i. 2, p. 1-53, 2004.

CABEDO, J. D; TIRADO, J. M. The disclosure of risk in financial statements. Accounting Forum, v. 28, i. 2, p. 181-200, 2003.

CARDOSO, R. L. et al. Análise da regulação da contabilidade à luz da teoria tridimensional do direito de Miguel Reale. Revista Universo Contábil, Blumenau, v. 6, n. 1, p. 6-27, jan./mar. 2010.

\section{COMITÊ DE PRONUNCIAMENTOS CONTÁBEIS. Pronunciamento Técnico} CPC 40 (R1) - Instrumentos financeiros: evidenciação. Jun. 2012. Disponível em: $<$ http://www.cpc.org.br/CPC/DocumentosEmitidos/Pronunciamentos/Pronunciamento?Id=71>. Acesso em: 24 out. 2014.

COSENZA, J. P.; LAURENCEL, L. C. Um olhar sobre a harmonização contábil no Brasil à luz da teoria Habermasiana. Revista de Informação Contábil, v. 5, n. 4, p. 79-103, out./dez. 2012.

DANTAS, J. A. et al. Determinantes do grau de evidenciação de risco de crédito pelos bancos brasileiros. Revista Contabilidade e Finanças, São Paulo, v. 21, n. 52, p. 1-27, jan./abr. 2010.

DOBLER, M. Incentives for risk reporting: a discretionary disclosure and cheap talk approach. The International Journal of Accounting, v. 43, i. 2, p. 184-206, 2008.

ELSHANDIDY, T.; FRASER, I.; HUSSAINEY, K. Aggregated, voluntary, and mandatory risk disclosure incentives: evidence from UK FTSE all-share companies. International Review of Financial Analysis, v. 30, p. 320-333, 2013. 
ELZAHAR, H.; HUSSAINEY, K. Determinants of narrative risk disclosures in UK interim reports. The Journal of Risk Finance, v. 13, i. 2, p. 133-147, 2012.

EMM, E. E.; GAY, G. D.; LIN, C. M. Choices and best practice in corporate risk management disclosure. Journal of Applied Corporate Finance, v. 19, i. 4, p. 1728, 2007.

FERNANDES, B. V. R. Modelos de valores extremos e convencionais de VAR: nível de acurácia na previsão de risco de mercado nos países do G7 e BRICS. 2012. 159 p. Tese (Doutorado em Ciências Contábeis)-Programa Multi-institucional e Inter-Regional de Pós-Graduação em Ciências Contábeis (UnB/UFPB/UFRN), Brasília, DF, 2012.

GOULART, A. M. C. Evidenciação contábil do risco de mercado por instituições financeiras no Brasil. 2003. 202 p. Dissertação (Mestrado em Controladoria e Contabilidade)-Universidade de São Paulo, São Paulo, 2003.

GRAÇA, M. L. Divulgação do risco de mercado um caso de empresas portuguesas. 2012. Dissertação (Mestrado em Contabilidade)-Instituto Politécnico de Setúbal, Setúbal, Portugal, 2012.

GUZZO, E. J. et al. Disclosure dos fatores de risco de mercado - FRM: uma análise das maiores companhias abertas do Brasil. Gestão Contemporânea, Porto Alegre, v. 10, n. 14, p. 247-273, jul./dez. 2013.

HASSAN, M. K. Risk narrative disclosure strategies to enhance organizational legitimacy: evidence from UAE financial institutions. International Journal of Disclosure and Governance, v. 11, p. 1-17, 2014.

HODDER, L.; KOONCE, L.; MCANALLY, M. L. SEC market risk disclosures: implications for judgment and decision making. Accounting Horizons, v. 15, i. 1, p. 49-70, 2001.

HUNZIKER, S. The disclosure of market risk information under IFRS 7 evidence from Swiss listed non-financial companies. IFZ Working Paper, v. 20, p. 1-18, 2013.

JENSEN, M. C.; MECKLING, W. H. Theory of the firm: managerial behavior, agency costs and ownership structure. Journal of Financial Economics, v. 3, i. 4, p. 305-360, 1976. 
KIRCH, G.; LIMA, J. B. N.; TERRA, P. R. S. Determinantes da defasagem na divulgação das demonstrações contábeis das companhias abertas brasileiras. Revista Contabilidade e Finanças, São Paulo, v. 23, n. 60, p. 173-186, set./dez. 2012.

LINSLEY, P. M.; SHRIVES, P. J. Risk reporting: a study of risk disclosures in the annual reports of UK companies. The British Accounting Review, v. 38, p. 387404, 2006.

MAPURUNGA, P. V. R.; PONTE, V. M. R.; HOLANDA, A. P. Fatores explicativos da aderência das firmas brasileiras ao disclosure relativo às informações por segmento. Enfoque: Reflexão Contábil, v. 33, n. 1, p. 53-69, jan./abr. 2014.

MARTINS, E. et al. Manual de contabilidade societária: aplicável a todas as sociedades de acordo com as normas internacionais e do CPC. 2. ed. São Paulo: Atlas, 2013.

MARTINS, O. S.; PAULO, E. A probabilidade de negociação com informação privilegiada no mercado acionário brasileiro. Revista Brasileira de Finanças, Rio de Janeiro, v. 11, n. 2, p. 249-280, jun. 2013.

MIIHKINEN, A. What drives quality of firm risk disclosure? The impact of a national disclosure standard and reporting incentives under IFRS. The International Journal of Accounting, v. 47, i. 4, p. 437-468, 2012.

MODIGLIANI, F.; MILLER, M. H. The cost of capital, corporation finance, and the theory of investment. The American Economic Review, v. 48, i. 3, p. 261-297, 1958.

MURCIA, F. D. Fatores determinantes do nível de disclosure voluntário de companhias abertas no Brasil. 2009. 182 p. Tese (Doutorado em Ciências Contábeis)Universidade de São Paulo, São Paulo, 2009.

OTHMAN, R.; AMEER, R. Market risk disclosure: evidence from Malaysian listed firms. Journal of Financial Regulation and Compliance, v. 17, i. 1, p. 57-69, 2009.

PINHO, G. A. et al. Associação entre risco e evidenciação de instrumentos financeiros no Brasil. In: CONGRESSO DA ASSOCIAÇÃO NACIONAL DE PROGRAMAS DE PÓS-GRADUAÇÃO EM CIÊNCIAS CONTÁBEIS, 8., 2014, Rio de Janeiro. Anais... Rio de Janeiro, 2014. 
PUTRI, N. K.; AROFAH, T. The impact of risk report formats on investment analyst decisions: an experimental case from Indonesia. Asian Academy of Management Journal of Accounting and Finance, v. 9, i. 1, p. 89-112, 2013.

SAVVIDES, S. C.; SAVVIDOU, N. Market risk disclosures of banks: a cross-country study. International Journal of Organizational Analysis, v. 20, i. 4, p. 379405, 2012.

SHINZATO, J. M. O uso de instrumentos financeiros e o nível de evidenciação qualitativa e quantitativa nas demonstrações contábeis de empresas não financeiras, no contexto das IFRS. 2011. 149 p. Dissertação (Mestrado em Ciências Contábeis e Financeiras)-Pontifícia Universidade Católica de São Paulo, São Paulo, 2011.

SILVA, A. M. M. Divulgação do risco: um caso de empresas do sector do retalho alimentar. 2012. 77 p. Dissertação (Mestrado em Contabilidade)-Instituto Politécnico de Setúbal, Setúbal, Portugal, 2012.

SOARES, E. R.; GALDI, F. C. Relação dos modelos DuPont com o retorno das ações no mercado brasileiro. Revista Contabilidade e Finanças, São Paulo, v. 22, n. 57, p. 279-298, set./dez. 2011.

WELKER, M. Disclosure policy, information asymmetry, and liquidity in equity markets. Conlemporary Accoiwnni; Research, v. 11, i. 2, p. 801-827, 1995.

\section{Como citar este artigo:}

\section{ABNT}

COSTA, Benedito Manoel do Nascimento; LEAL, Paulo Henrique; PONTE, Vera Maria Rodrigues. Determinantes da divulgação de informações de risco de mercado por empresas não financeiras. RACE, Revista de Administração, Contabilidade e Economia, Joaçaba: Ed. Unoesc, v. 16, n. 2, p. 729-756, maio/ago. 2017. Disponível em: <http://editora.unoesc.edu.br/index.php/race>. Acesso em: dia/mês/ano.

APA

Costa, B. M. do N., Leal, P. H., \& Ponte, V. M. R. (2017). Determinantes da divulgação de informações de risco de mercado por empresas não financeiras. RACE, Revista de Administração, Contabilidade e Economia, 16(2), 729-756. Recuperado em dia/mês/ano, de http://editora.unoesc.edu.br/index.php/race 
